\title{
Pairs of Bi-Cubic Surface Constructions Supporting Polar Connectivity
}

\author{
Ashish Myles ${ }^{\text {a,* }}$ Kestutis Karčiauskas ${ }^{b}$ Jörg Peters ${ }^{\text {a }}$

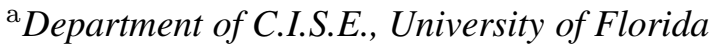 \\ ${ }^{\mathrm{b}}$ Department of Mathematics and Informatics, Vilnius University
}

\begin{abstract}
Surface constructions of polynomial degree $(3,3)$ come in four flavours that complement each other: one pair extends the subdivision paradigm, the other the NURBS patch approach to free-form modeling.

The first pair, Catmull-Clark (Catmull and Clark, 1978) and Polar subdivision (Karčiauskas and Peters, 2007) generalize bi-cubic subdivision: While Catmull-Clark subdivision is more suitable where few facets join, Polar subdivision nicely models regions where many facets join as when capping extruded features. We show how to easily combine (the meshes of) these two generalizations of bi-cubic spline subdivision.

The second pair of surface constructions with a finite number of patches consists of PCCM (Peters, 2000) for layouts where Catmull-Clark would apply and a singularly parameterized NURBS patch for polar layout. A novel analysis shows the latter to yield a $C^{1}$ surface with bounded curvatures.
\end{abstract}

Key words: bounded curvature, bi-cubic, $C^{1}$, tensor-product, finite, B-spline, NURBS, mesh refinement, PCCM, Catmull-Clark, subdivision

\section{Motivation, Literature and Overview}

While the mesh-based subdivision representation yields an intuitive visualization for interactive modeling, surfaces constructed from finitely many NURBS patches are preferred in CAD packages and convenient for GPU implementations. All four

*

Email addresses: amyles@cise.ufl . edu (Ashish Myles), kestutis.karciauskas@mif.vu.lt (Kestutis Karčiauskas), jorg@cise.ufl. edu (Jörg Peters).

URL: http: / / www. cise.ufl. edu/ jorg (Jörg Peters). 
Table 1

\begin{tabular}{l|ll}
\hline layout & sprocket-like & polar \\
\hline subdivision & Catmull-Clark & bi-cubic polar \\
finite & PCCM & new (Section 3)
\end{tabular}

Bi-cubic surface constructions: (Catmull and Clark, 1978), (Karčiauskas and Peters, 2007), (Peters, 2000) (See also Figure 11).

piecewise bi-cubic surface types of Table 1 are $C^{2}$ almost everywhere and $C^{1}$ otherwise and, because they are a finite number of bi-cubic splines in 'regular regions', they are compatible in the sense that they can be used in combination to model different regions of an object.

Generalized bi-cubic subdivision is traditionally associated with $\mathrm{CC}$ subdivision (Catmull and Clark, 1978). CC subdivision is popular in graphics due to its close relation to the industry standard bi-cubic tensor-product splines or NURBS. However, $\mathrm{CC}$ subdivision is prone to generating saddle points in the limit even though the control net is designed for convex shape (Karčiauskas et al., 2004). This has motivated the improvements in (Levin, 2006; Ginkel and Umlauf, 2006; Augsdörfer et al., 2006). A second problem of CC subdivision is that, where many facets join or where features are extruded, the resulting shape can be poor and show unintended ripples (see e.g. Figure 1). Trying to suppress such macroscopic ripples arising in the first subdivision step motivated the global shape optimization (Halstead et al., 1993). In (Karčiauskas et al., 2006), it was argued that both ripple and saddle problems can be more simply resolved by switching to a polar layout of facets (Figure 1). A neighborhood of a central high-valence vertex (Figure 2) has polar layout if the vertex is surrounded by one layer of triangles while the remaining mesh consists of quadrilaterals, with always four facets joining at each non-central vertex. Polar layout naturally appears in the design of surfaces of revolution. Recently, a sim-

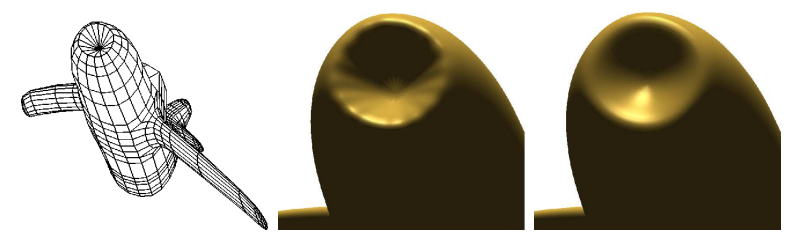

Fig. 1. (left) Airplane with polar nose. (middle) Catmull-Clark subdivision generates ripples, whereas (right) polar subdivision looks natural.
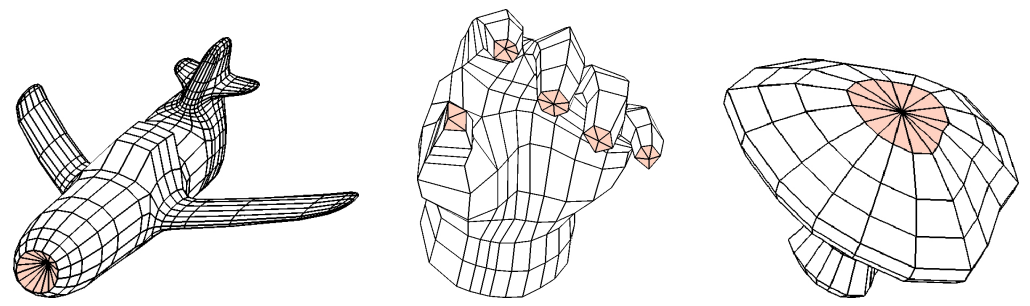

Fig. 2. Augmenting Catmull-Clark meshes with polar vertices: (left) nose of airplane, (middle) tips of fingers, (right) mushroom top. 

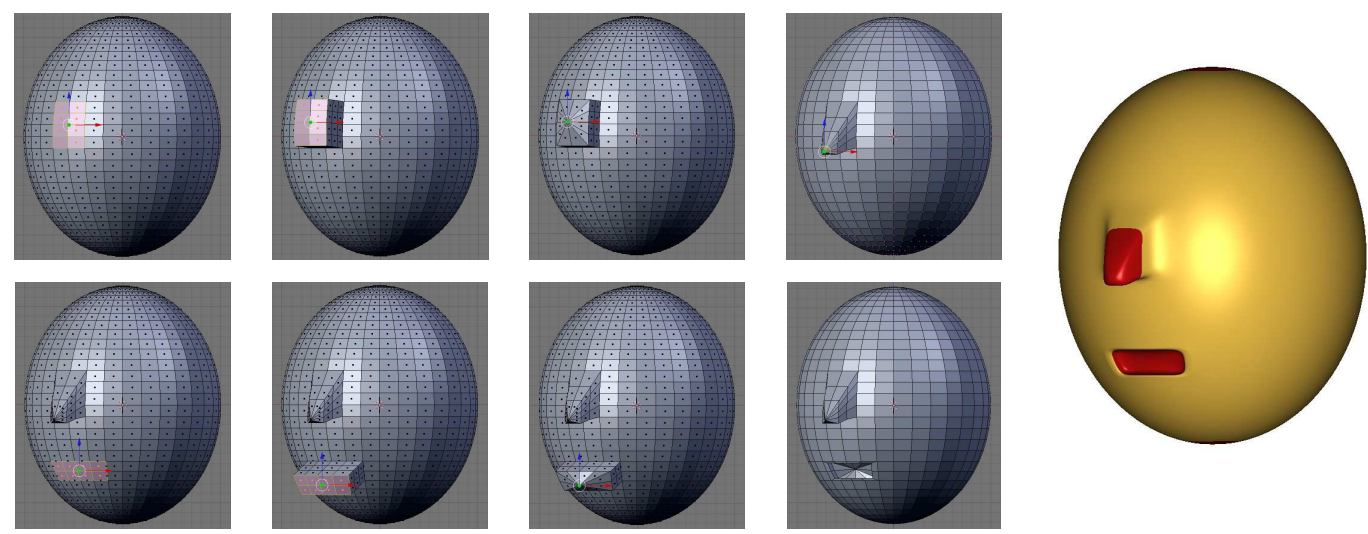

Fig. 3. Polar vertices simplify modeling. (left to right, top to bottom) A nose and a mouth are extruded (introducing valence 5 vertices at the base corners) and capped with a high-valent polar vertex. (right) Final surface.

ple generalization of bi-cubic subdivision to polar layouts was proposed, bi-cubic Polar (BP) subdivision (Karčiauskas and Peters, 2007). Polar meshes can also be refined using the more general tool of Quad-tri subdivision (Stam and Loop, 2003; Schaefer and Warren, 2005); but BP subdivision (Karčiauskas and Peters, 2007) is preferable for polar layout because it generates a finite number of bi-cubic patches in the transition from a quad to a triangle facet while Quad-tri subdivision creates infinitely many patches, half of them three-sided. Also for the polar layout, Jet subdivision (Karčiauskas et al., 2006) generates $C^{2}$ surfaces with good curvature distribution. But the Jet subdivision is more complex and results in surfaces of degree $(6,5)$; and are therefore outside of our focus on bi-cubic schemes. Similarly, replacing CC meshes by those generated in (Levin, 2006) is compatible with Polar subdivision but outside our focus.

The approach for combining BP subdivision and CC subdivision meshes is to use $\mathrm{CC}$ subdivision except for special rules where the mesh has a polar layout. This has the advantage that any input mesh admissible for CC subdivision can be handled by the resulting combined subdivision. In effect, the polar submeshes will be temporarily split off from the remaining mesh so that the same code base as for $\mathrm{CC}$ subdivision can be used and just one special subroutine for polar submeshes is added to improve the surface quality. The resulting surface pieces match exactly at their interfaces where they represent the same bi-cubic polynomial spline patches.

Our approach for complementing a finite bi-cubic spline construction with a finite polar construction is analogous and compatible with PCCM (Peters, 2000). We interpret, after a few steps, the Polar subdivision control mesh as the control net of a NURBS surface. This single polynomial NURBS patch has an edge collapsed to a point and only the NURBS coefficients next to it have to be adjusted. The result is a $C^{1}$ surface with bounded curvature. Due to the growth of the regular mesh and the boundedness of the construction near irregular mesh points, refining the input mesh allows switching from the subdivision mesh to the NURBS representation 
and vice versa with an error decreasing with the subdivision level.

In Section 2, we review and extend BP subdivision and show how to apply it in the context of meshes of arbitrary connectivity. In Section 3, we construct a single singularly parameterized NURBS patch for completing a polar cap. The cap's smoothness is analyzed with a novel approach.

\subsection{Definitions}

A control net or mesh is an embedding of a graph in $\mathbb{R}^{3}$ with vertices identified as points and connectivity indicated by line segments. The graph is assumed to have the connectivity of a 2-manifold. The number of neighbors of a vertex is called the valence of the vertex. It is denoted by $n$. The $i$-link of a vertex consists of points that can be reached by traversing a shortest path of $i$ edges. The 0-link consists only of the vertex itself. The 1-link consists of the direct neighbors. The $i$-layer of a vertex $\mathbf{v}$ is the collection of faces, whose closest vertex to $\mathbf{v}$ is in $\mathbf{v}$ 's $i$-link. The 0 -layer of $\mathbf{v}$ consists of all its incident faces.

A polar vertex is one whose 0-layer consists only of triangles (see Figure 2). A polar structure consists of a polar vertex of valence $n \geq 6$ whose 1-layer and 2layer consist only of quadrilateral facets (quads) and such that the vertices of the 1- and 2-link are 4-valent (see Figure 2). We will not need to consider polar rules for boundaries since such boundaries are modeled by the complementary CatmullClark or PCCM rules. A polar structure makes the term polar layout precise.

\section{Compatible polar mesh refinement and Subdivision}

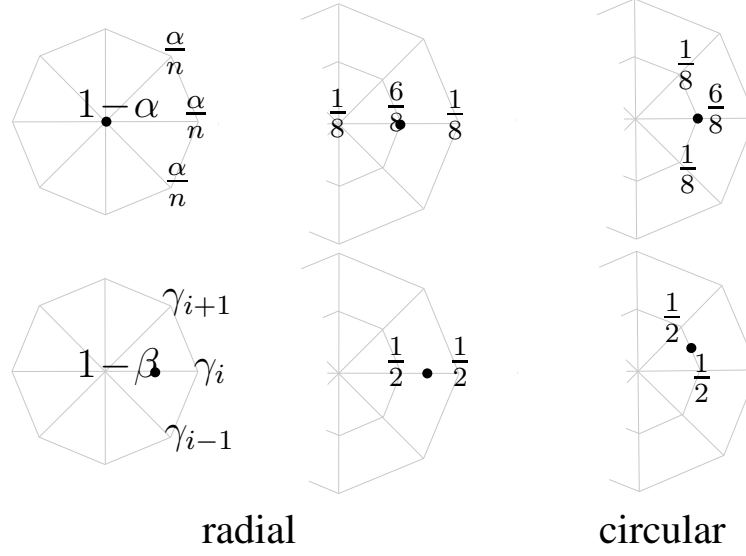

Fig. 4. Refinement stencils for Polar subdivision (Karčiauskas and Peters, 2007). (left) Radial subdivision at the polar vertex, (middle) radial subdivision everywhere else, (right) circular subdivision everywhere else. 


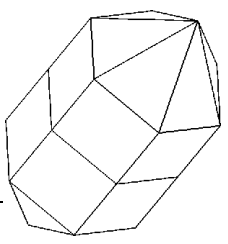

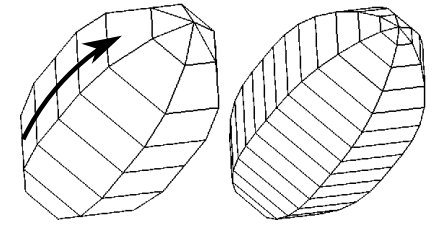

radial

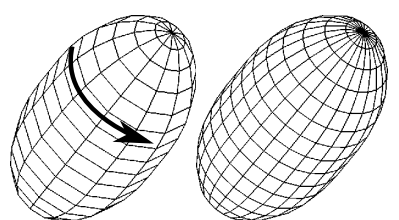

circular

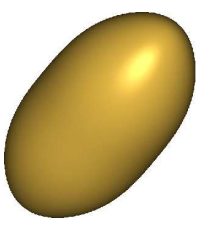

Fig. 6. Mesh refinement preserving the limit surface of BP subdivision. From left to right: original mesh, two applications of radial subdivision, followed by two applications of circular subdivision, and limit surface.

Compatible polar mesh refinement is a straightforward approach to generating a consistently $k$-times subdivided mesh when the Catmull-Clark mesh is augmented with polar structures. For a mesh with the latitude-longitude connectivity, BP subdivision applies cubic spline refinement only in the longitudinal (radial) but not in the latitudinal (circular) direction. For the polar vertex and its 1-link, the stencil weights for Figure 4, left, are given as

$$
\begin{gathered}
\alpha:=\beta-\frac{1}{4}, \quad \beta:=\frac{5}{8}, \quad c_{n}^{k}:=\cos \left(\frac{2 \pi k}{n}\right), \\
\gamma_{k}:=\frac{1}{n}\left(\beta-\frac{1}{2}+\frac{5}{8} c_{n}^{k}+\left(c_{n}^{k}\right)^{2}+\frac{1}{2}\left(c_{n}^{k}\right)^{3}\right),
\end{gathered}
$$

where $n$ is the valence of the polar vertex. The analysis in (Karčiauskas and Peters, 2007) shows that, for $n \geq 6$, this radial subdivision results in an everywhere $C^{2}$ surface except at the central limit point. At the central point the surface is $C^{1}$ with bounded curvature. Moreover, the ripple and saddle artifacts of Catmull-Clark subdivision do not appear.

Evidently, as illustrated in Figure 5, purely radial refinement results in a mismatch or a mesh with $\mathrm{T}$ corners at the transition to Catmull-Clark subdivision since Catmull-Clark subdivision simultaneously subdivides radially and circularly. In order to leverage and preserve the good results of radial subdivision and still display a consistent control net after $k$ steps, we proceed as illustrated in Figure 6: we do not alternate radial and circular subdivisions in the $k$ steps but apply the following compatible polar mesh refinement.

(a) Apply $k$ steps of radial subdivision and save the polar structure at level $k$ in case we continue subdivision later.

(b) Apply $k$ circular subdivision steps.

We base any continued refinement on the saved polar structure. While step (a) preserves the valence, simplifying implementation, applying step (b) a posteriori amounts to knot insertion that does not change the surface. Therefore the analysis of 
(Karčiauskas and Peters, 2007) applies. By contrast, the equally obvious approach of alternating radial and circular subdivision creates local curvature fluctuations and requires a new analysis. So, compatible polar refinement is preferable.

Since we only locally improve CC subdivision, all input meshes suitable for CC subdivision are admissible for the combined BP and CC subdivision. In particular, global boundaries and $n$-sided facets are covered by CC rules. A designer wanting to treat a vertex with Polar subdivision, has to configure its neighborhood as a polar structure (Section 1.1). A typical scenario is a cylindrical extrusion surrounded by 5 -sided facets (see Figure 3). Given an input mesh and the maximal subdivision level $k$, generalized bi-cubic subdivision for Catmull-Clark meshes augmented with polar structures is straightforward (see Figure 7):

1. Split off polar structures: Copy all the polar structures and remove polar vertices from the input mesh.

2. Subdivide polar structures: For each polar structure,

(a) subdivide $k$ times radially, and then

(b) subdivide $k$ times in the circular direction.

3. Subdivide the remaining mesh: Apply $k$ steps of Catmull-Clark subdivision to the mesh without the polar vertices.

4. Merge results: Drop the boundary facets of the meshes subdivided in steps 2 and 3 and join them by identifying the resulting geometrically identical boundary vertices.

By splitting the mesh into overlapping pieces, we introduced new boundaries in addition to any global boundaries of the input mesh. Subdivision steps 2 and 3 deal with these boundaries by dropping the vertices that do not have enough neighbors to apply the regular rules.

If a designer placed polar vertices too close together, or did not separate extraordinary limit points of Catmull-Clark subdivision, or created polar points of low valence, then applying step 3 and standard bi-cubic subdivision also in the 0-layer of the polar vertex (by interpreting triangular facets as degenerate quadrilaterals) doubles the valence and separates extraordinary limit points (see Figure 8). For this one initial step, computing the polar vertex with weight $\alpha:=\frac{3}{8(1-\beta)}-\frac{1}{2}$ yields

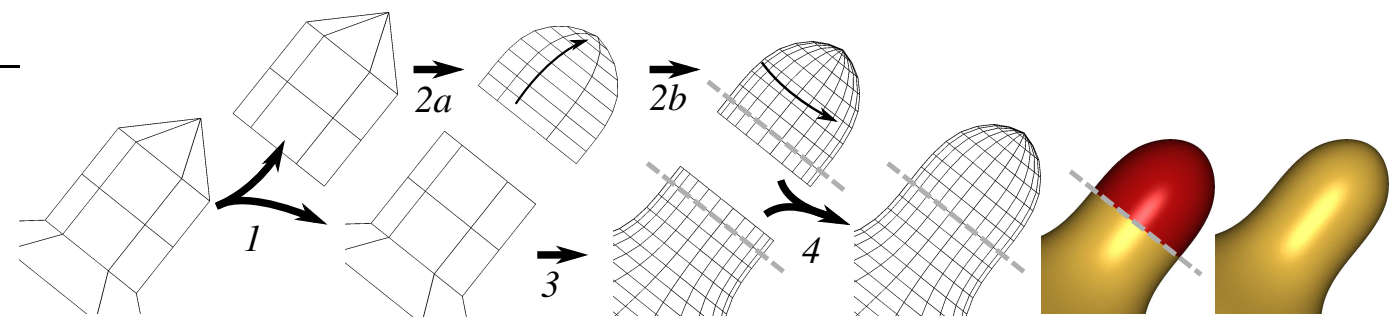

Fig. 7. Generalized bi-cubic subdivision steps. (1) Separating the input mesh. (2) Subdividing the polar structure (2a) radially then (2b) circularly. (3) Subdividing the remainder. (4) Joining the refined meshes after removal of overlapping facets. (right) The limit surface. 

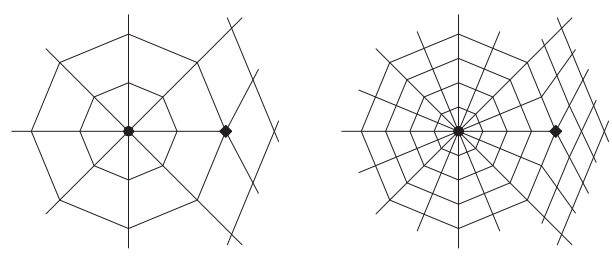

Fig. 8. Separating Catmull-Clark and polar extraordinary limit points.

good shapes for a range of $\beta$. We suggest $\beta:=\frac{1}{2}$ and $\alpha=\frac{1}{4}$.

\section{Finite bi-cubic constructions}
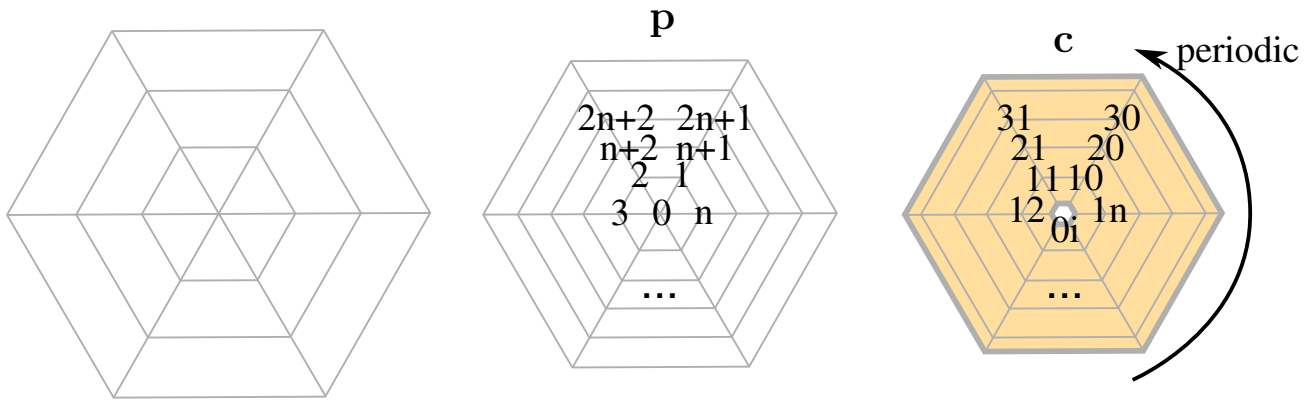

Fig. 9. Finite polar capping by a periodic singular NURBS patch. (left) Initial polar structure, (middle) polar mesh with vertices $\mathbf{p}_{i}$ after one subdivision; (right) bi-cubic NURBS representation with control points $\mathbf{c}_{i j}$ : the collapsed center $\mathbf{c}_{0 i}$ and the exterior boundary are associated with 4-fold knots.

A complementary generalization of the bi-cubic setting is to model areas deviating from the tensor-product setting by a few bi-cubic NURBS patches. Since PCCM (Peters, 2000) gives a construction for Catmull-Clark layouts, we focus here on constructing a single bi-cubic spline for the polar structure. Just as PCCM this yields a finite bi-cubic surface that is $C^{2}$ almost everywhere and $C^{1}$ otherwise. On input of a polar structure, the finite bi-cubic NURBS construction has the following steps (cf. Figures 9 and 10).

(i) (recommended for better shape) Subdivide the polar structure. Subdivide radially, twice for elongated examples like tips of fingers. The resulting mesh $\mathbf{p}$ is labeled as in Figure 9, middle.

(ii) Convert the polar structure to a spline mesh. Initialize $\mathbf{c}_{i j}:=\mathbf{p}_{(i-1) n+j+1}$ for $i>1$ and $j=0, \ldots, n-1$. Both $u$ and $v$ knot sequences are uniform. The circular direction with parameter $u$ is periodic.

(iii) Interpolate the extraordinary limit point of $B P$ subdivision. For $i=0, \ldots, n-$ 1 , set

$$
\mathbf{c}_{0 i}:=\eta \mathbf{p}_{0}+(1-\eta) \frac{1}{n} \sum_{j=1}^{n} \mathbf{p}_{j}, \eta:=\frac{4(1-\beta)}{3}
$$




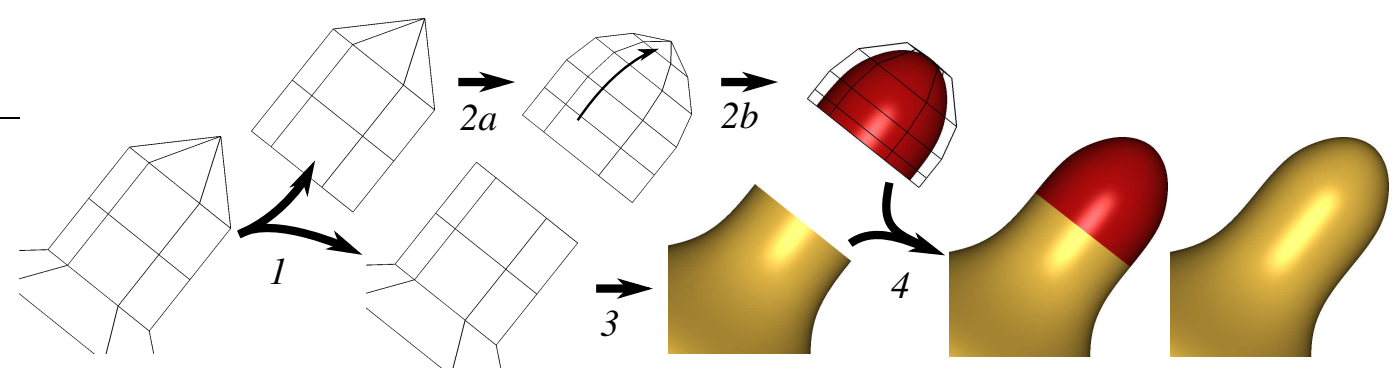

Fig. 10. Finite polar capping. (1) Separating the input mesh. (2a) Subdividing the polar structure radially. (2b) Projecting the 1-link to the limit tangent, knot insertion, and re-interpretation as a periodic B-spline. (3) PCCM. (4) Joining the surfaces. (right) Surface consisting of finitely many bi-cubic patches.

and change the start of the radial knot sequence to a 4-fold knot associated with $\mathbf{c}_{0 i}$.

(iv) Match the limit normal of BP subdivision. We project the neighbors of the central point into a common plane. For $i=0, \ldots, n-1$,

$$
\begin{aligned}
\mathbf{c}_{1 i} & :=\mathbf{c}_{0 i}+2 \sigma \sum_{j=0}^{n-1} \Gamma_{j-i} \mathbf{p}_{j+1}, \quad \sigma_{\text {default }}:=\frac{3}{4} \\
\Gamma_{k} & :=\frac{1}{n} \cos \left(\frac{2 \pi k}{n}\right)
\end{aligned}
$$

The projection of the spline coefficients does not alter the inherent $C^{2}$ continuity of the spline apart from the singularity at the extraordinary limit point; and the projection maps all radial tangents into the same plane with normal direction $\left(\mathbf{c}_{11}-\mathbf{c}_{00}\right) \times\left(\mathbf{c}_{12}-\mathbf{c}_{00}\right)$ at the extraordinary limit point.

(v) (optional) Additional knot insertion. It is common to have cubic NURBS patches with four-fold end knots. Knot insertion at the outer boundary yields e.g. $0,0,0,0,1,2, \ldots, m-1, m, m, m, m$ for the radial knots. The circular knot sequence remains uniform due to periodicity.

Figure 12 and 13, right, show examples of the NURBS construction. Due to the common interpolated control vertex $\mathbf{c}_{00}$ that represents a collapsed edge $\mathbf{c}_{0 i}:=\mathbf{c}_{00}$, $i=0, \ldots, n-1$, the spline surface is $C^{0}$ but singularly parametrized. Since singularly parametrized surfaces are commonly used in CAD applications, such packages handle and display the NURBS patch without problems. However, singularly parametrized surfaces are tricky to analyze (Neamtu and Pfluger, 1994; Peters, 1991; Reif, 1995a; Bohl and Reif, 1997; Reif, 1998). The classical approach is an algebraic reparametrization of the surface in the singular point. We use a different approach that only becomes natural due to improved understanding of subdivision surfaces: we derive a projected bi-cubic subdivision (pbs) that traces out the same surface as the NURBS patch. First we document, what is intuitively clear, namely that an arbitrary B-spline with one end collapsed to an interpolated vertex is generically not $C^{1}$. 
Lemma 1 Without the projection step (iv), the singular NURBS patch construction in Section 3 is generically not $C^{1}$.

Proof We focus on the $i$-links of the polar vertex $\mathbf{c}_{00}$ for $i=0,1,2,3$, and initialize for subdivision level $m=0, \mathbf{c}_{k i}^{m}:=\mathbf{c}_{i j}$ for $j=0, \ldots, n-1, k=0,1,2,3$. For

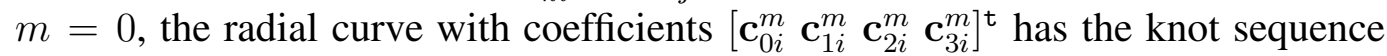
$0,0,0,0,1,2,3,4$ and standard subdivision amounts to inserting knots at $1 / 2$ and $3 / 2$ into the radial curve representation, i.e. to subdivision equations

$$
\left[\begin{array}{l}
\mathbf{c}_{0 i}^{m+1} \\
\mathbf{c}_{1 i}^{m+1} \\
\mathbf{c}_{2 i}^{m+1} \\
\mathbf{c}_{3 i}^{m+1}
\end{array}\right]=\left[\begin{array}{cccc}
1 & 0 & 0 & 0 \\
1 / 2 & 1 / 2 & 0 & 0 \\
0 & 3 / 4 & 1 / 4 & 0 \\
0 & 3 / 16 & 11 / 16 & 1 / 8
\end{array}\right]\left[\begin{array}{l}
\mathbf{c}_{0 i}^{m} \\
\mathbf{c}_{1 i}^{m} \\
\mathbf{c}_{2 i}^{m} \\
\mathbf{c}_{3 i}^{m}
\end{array}\right]
$$

The subdivision matrix of the NURBS patch combines $n$ curve subdivision matrices, and its trivial spectral analysis shows an $n$-fold eigenvalue of 1 that corresponds to a single leading eigenvalue by the standard approach of distributing the contribution $\mathbf{c}_{0 i}=\mathbf{c}_{00}$ evenly with weight $1 / n$. However, there are also $n$ subdominant eigenvalues of size $1 / 2$ that contradict the uniqueness of a tangent plane for generic data. In general, such a scheme is not $C^{1}$.

Construction step (iv) in Section 3 maps the 1-link into a unique plane. To use this projection in the analysis, we say the 1-link vertices $\mathbf{c}_{1 i}$ are in oval position if there exist two linearly independent vectors $\mathbf{e}_{1}$ and $\mathbf{e}_{2}$ such that

$$
\mathbf{c}_{1 i}=\mathbf{c}_{00}+\mathbf{e}_{1} \cos (2 \pi i / n)+\mathbf{e}_{2} \sin (2 \pi i / n) .
$$

That is, the 1-link is equally distributed on an oval, the affine projection of a circle centered at the extraordinary limit point $\mathbf{c}_{00}$, and lies in the plane containing $\mathbf{c}_{00}$ and spanned by $\mathbf{e}_{1}$ and $\mathbf{e}_{2}$. We observe that,

- if the control points $\mathbf{c}_{1 i}^{m}$ are in oval position, row two of Equation 4 assigns $\left(\mathbf{c}_{00}^{m}+\mathbf{c}_{1 i}^{m}\right) / 2$ to $\mathbf{c}_{1 i}^{m+1}$ so that the points $\mathbf{c}_{1 i}^{m+1}$ are also in oval position.

- Equation 3, in the form

$$
\mathbf{c}_{1 i}^{m} \leftarrow \mathbf{c}_{00}^{m}+2 \sigma \sum_{j=0}^{n-1} \Gamma_{j-i} \mathbf{c}_{1 j}^{m}
$$

maps a 1-link $\mathbf{c}_{1 i}$ into oval position. Applying projection (6) for $\sigma=1$ on both sides of Equation 5 shows that a 1-link in oval position remains unchanged under the projection.

We now define the projected bi-cubic subdivision (pbs) scheme to be the standard bi-cubic subdivision but with the second row in (4) modified by applying the projection (6):

$$
\mathbf{c}_{1 i}^{m+1}=\frac{1}{2} \mathbf{c}_{0 i}^{m}+\frac{1}{2}\left(\mathbf{c}_{0 i}^{m}+2 \sum_{j=0}^{n-1} \Gamma_{j-i} \mathbf{c}_{1 j}^{m}\right)=\mathbf{c}_{0 i}^{m}+\sum_{j=0}^{n-1} \Gamma_{j-i} \mathbf{c}_{1 j}^{m} .
$$


Lemma 2 The singular NURBS patch defined in Section 3 is identical to the limit surface generated when pbs is applied to its control points.

Proof By construction of the NURBS patch, the 1-link $\mathbf{c}_{1 j}^{0}$ is in oval position. For a 1-link $\mathbf{c}_{1 j}$ in oval position, Equation 7 and row two of Equation 4 yield identical new 1-links.

A standard analysis of the mesh with the $\mathbf{c}_{0 i}$ interpreted as a single point shows pbs to generate $C^{1}$ surfaces with bounded curvature, with the usual caveat that control points should be generic (Reif and Peters, 2005, p 164).

Lemma 3 For generic input data, surfaces generated by pbs are $C^{1}$ and have bounded curvature.

Proof We split the contribution of $\mathbf{c}_{00}$ evenly amongst the $\mathbf{c}_{0 i} i \in 0, \ldots, n-1$ and write the pbs circulant matrix as

$$
\left[\begin{array}{c}
\vdots \\
\mathbf{c}_{0 i}^{m+1} \\
\mathbf{c}_{1 i}^{m+1} \\
\mathbf{c}_{2 i}^{m+1} \\
\mathbf{c}_{3 i}^{m+1} \\
\vdots
\end{array}\right]=\left[\begin{array}{cccc}
A_{0} & A_{1} & \ldots & A_{n-1} \\
A_{n-1} & A_{0} & \ldots & A_{n-2} \\
\vdots & & \ddots & \vdots \\
A_{1} & \ldots & A_{n-1} & A_{0}
\end{array}\right]\left[\begin{array}{c}
\vdots \\
\mathbf{c}_{0 i}^{m} \\
\mathbf{c}_{1 i}^{m} \\
\mathbf{c}_{2 i}^{m} \\
\mathbf{c}_{3 i}^{m} \\
\vdots \\
\end{array}\right]
$$

where for $i>0$,

$$
A_{0}:=\left[\begin{array}{cccc}
1 / n & 0 & 0 & 0 \\
1 / n & \Gamma_{0} & 0 & 0 \\
0 & 3 / 4 & 1 / 4 & 0 \\
0 & 3 / 16 & 11 / 16 & 1 / 8
\end{array}\right], A_{i}:=\left[\begin{array}{cccc}
1 / n & 0 & 0 & 0 \\
1 / n & \Gamma_{i} & 0 & 0 \\
0 & 0 & 0 & 0 \\
0 & 0 & 0 & 0
\end{array}\right]
$$

Under discrete Fourier transform, $\hat{A}_{i}:=\sum_{j=0}^{n-1} w^{i j} A_{j}, w^{i j}:=\exp (2 \pi i j \sqrt{-1} / n)$, the circulant system diagonalizes into $\operatorname{diag}\left(\hat{A}_{0}, \ldots, \hat{A}_{n-1}\right)$ where $\hat{\Gamma}_{i}:=\sum_{j=0}^{n-1} w^{i j} \Gamma_{j}$ and

$$
\hat{A}_{0}:=\left[\begin{array}{cccc}
1 & 0 & 0 & 0 \\
1 & \hat{\Gamma}_{0} & 0 & 0 \\
0 & 3 / 4 & 1 / 4 & 0 \\
0 & 3 / 16 & 11 / 16 & 1 / 8
\end{array}\right], \quad \hat{A}_{i}:=\left[\begin{array}{cccc}
0 & 0 & 0 & 0 \\
0 & \hat{\Gamma}_{i} & 0 & 0 \\
0 & 3 / 4 & 1 / 4 & 0 \\
0 & 3 / 16 & 11 / 16 & 1 / 8
\end{array}\right]
$$

The eigenvalues of $\hat{A}_{0}$ are $1, \hat{\Gamma}_{0}, 1 / 4,1 / 8$, and those of $\hat{A}_{i}$ are $0, \hat{\Gamma}_{i}, 1 / 4,1 / 8$. Since $\hat{\Gamma}_{0}=0$ and $\hat{\Gamma}_{1}=\hat{\Gamma}_{n-1}=1 / 2$, while $\hat{\Gamma}_{i}=0$ in all other cases, the subdominant eigenvalue is $\lambda:=1 / 2$ and corresponds to Fourier blocks $\hat{A}_{1}$ and $\hat{A}_{n-1}$. The right eigenvector of $\hat{A}_{1}$ corresponding to eigenvalue $1 / 2$ is $(0,1,3,6)$. Therefore the characteristic map $\mathbf{r}$ (labeled using the same indexing as $\mathbf{c}$ in Figure 9, right) has coefficients

$$
\left(\mathbf{r}_{0 i}, \mathbf{r}_{1 i}, \mathbf{r}_{2 i}, \mathbf{r}_{3 i}\right):=\left(\mathbf{0}, \mathbf{s}_{i}, 3 \mathbf{s}_{i}, 6 \mathbf{s}_{i}\right), \quad \mathbf{s}_{i}:=\left[\begin{array}{c}
\cos \left(\frac{2 \pi i}{n}\right) \\
\sin \left(\frac{2 \pi i}{n}\right)
\end{array}\right] .
$$




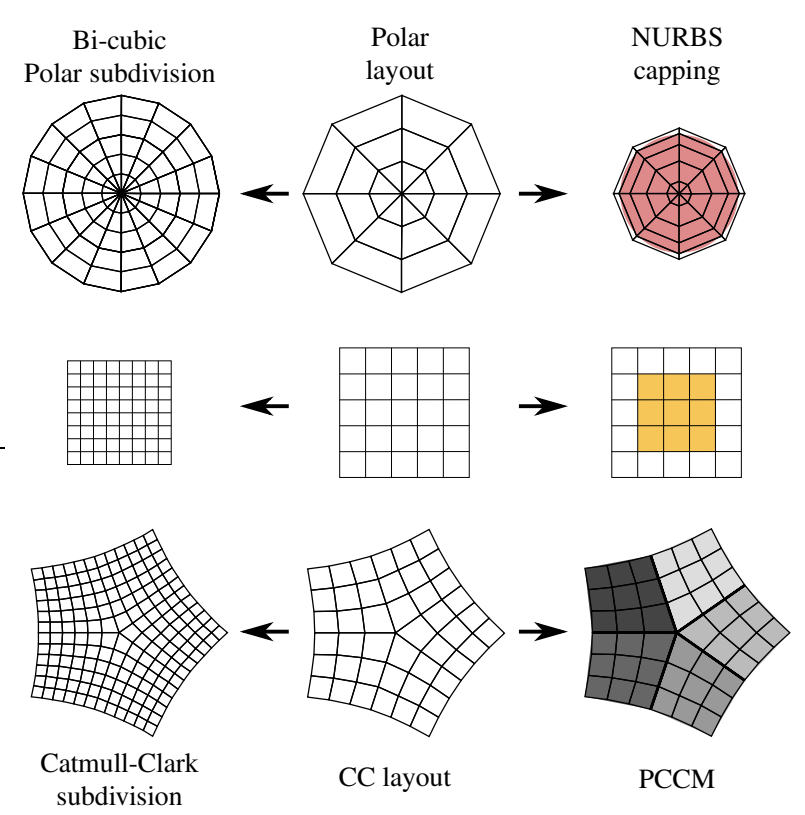

Fig. 11. Generalizations of standard bi-cubic B-splines (middle row) to quads with nonfour-valent vertices (bottom row) and polar vertices (top row), with mesh refinement rules (left column) or explicit spline patch constructions (right column).

The characteristic map is regular for arbitrary valences and satisfies the injectivity criterion of Theorem 3.5 of (Reif, 1995b), so that the limit surface is $C^{1}$ as claimed. The subsubdominant eigenvalue $\mu:=1 / 4=\lambda^{2}$ comes from independent Fourier blocks, indicating that it has equal algebraic and geometric multiplicity. Hence by Theorem 3.3 of (Peters and Reif, 2004), the surface has bounded curvature.

Lemma 2 and 3 imply the hoped-for theorem.

Theorem 4 For generic control nets, a NURBS patch constructed according to Section 3 is $C^{1}$ and has bounded curvature at the central point.

\section{Summary and Discussion}

All four surface types generalizing bi-cubic splines, shown in Figure 11 (and Table 1), are compatible with one another in that their transitions are identical bi-cubic splines. The resulting surfaces are piecewise bi-cubic, $C^{2}$ almost everywhere and $C^{1}$ at isolated points (curves in the case of PCCM). Both the subdivision and the NURBS construction give equally valid meaning to the input mesh created and manipulated by the designer. And, by increasing the subdivision level, the resulting surfaces can be made arbitrarily close to allow switching from one modeling paradigm to the other. The polar pieces of each approach can be implemented as a simple extension of existing modeling tools.

Since our finite polar spline generalization of bi-cubic splines consists of a single 

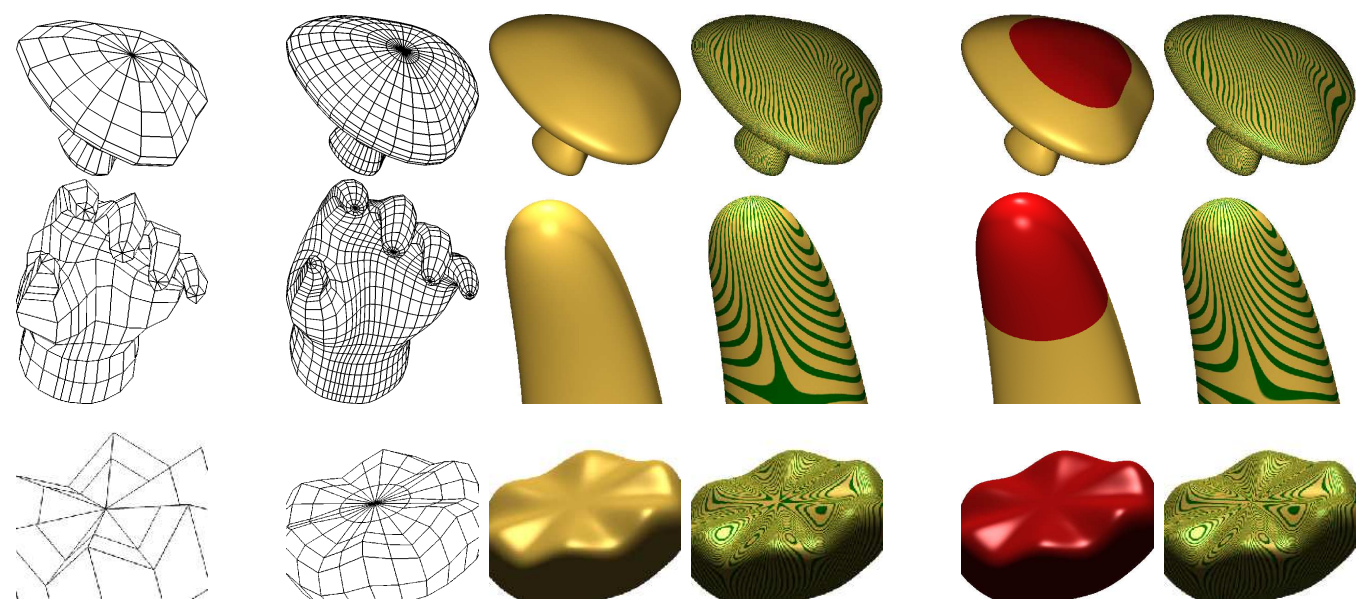

Fig. 12. Results. From left to right: control mesh, mesh after one subdivision, subdivision limit surface, highlight lines on subdivision surface, finite NURBS construction, finite NURBS construction highlight lines. In row two, we zoom in on one of the fingers. Row three illustrates a high-valent saddle.

NURBS patch, it is easy to add to existing CAD and animation modeling packages and is suitable for evaluation on the GPU (Krishnamurthy et al., 2007). The central singularity presents no problem for rendering since the explicit normal is known and the surface curvatures are bounded.

The analysis of this finite construction defines and uses another polar subdivision scheme, called pbs. This raises the question whether we could use pbs in place of BP subdivision and thereby obtain a unified finite-plus-subdivision representation. We consider pbs less practical since it has a large subdivision footprint, with special rules for every $i$-link for $i=0,1,2,3$. Moreover, the generating functions associated with the 1-link vertices are dependent and a special first step is required without which the convex hull property is not guaranteed.

BP subdivision augments the capabilities of existing Catmull-Clark implementations. The extension is particularly valuable for extruded features and naturally complements Catmull-Clark in regions of high-valence. While the smooth connection of the two surface types was never in doubt since they both reduce to bi-cubic splines. it is necessary (and simple) to define a compatible polar mesh refinement to minimally modify the existing infrastructure and add the shape and simplicity of BP subdivision.

\section{References}

Augsdörfer, U. H., Dodgson, N. A., Sabin, M. A., 2006. Tuning subdivision by minimising gaussian curvature variation near extraordinary vertices. Computer Graphics Forum (Proc. Eurographics) 25 (3), 263-272. 

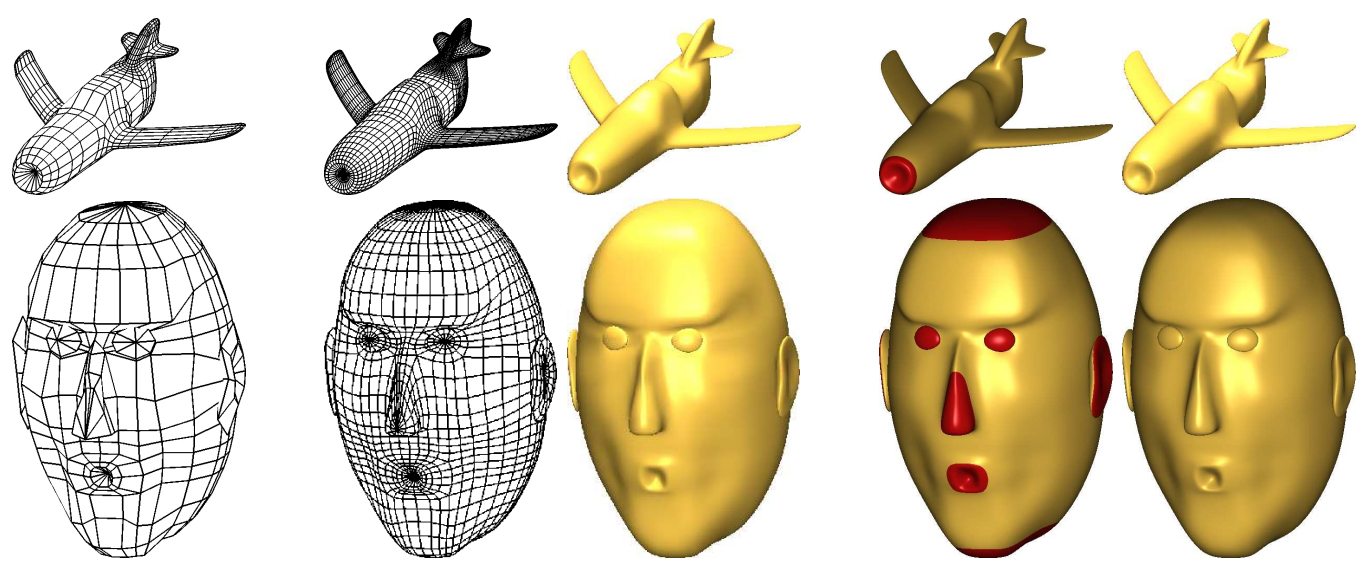

Fig. 13. Results. From left to right: control mesh, mesh after one subdivision, subdivision limit surface, polar regions, finite NURBS construction.
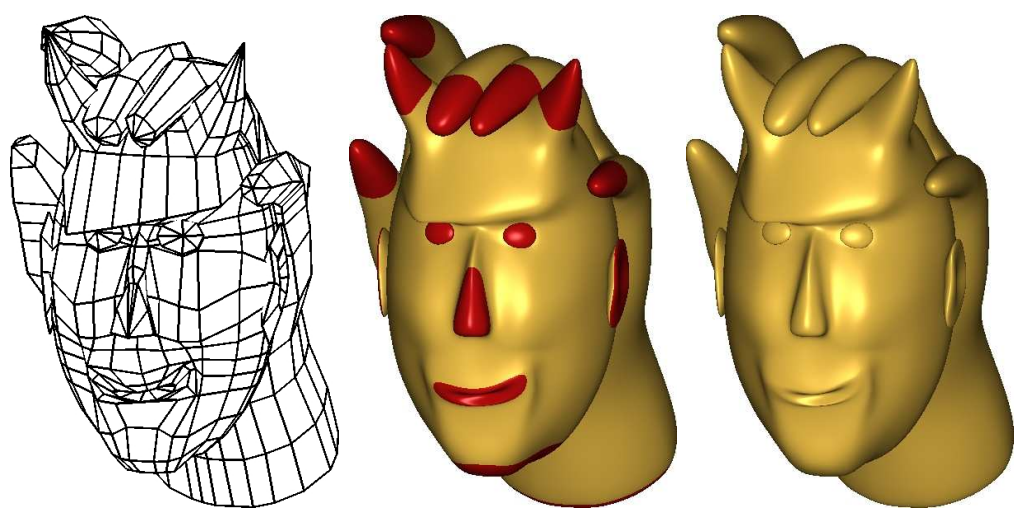

Fig. 14. Polar vertices help even in frightening scenarios.

Bohl, H., Reif, U., 1997. Degenerate Beźier patches with continuous curvature. Computer Aided Geometric Design 14 (8), 749-761.

Catmull, E., Clark, J., 1978. Recursively generated B-spline surfaces on arbitrary topological meshes. Computer Aided Design 10, 350-355.

Ginkel, I., Umlauf, G., 2006. Loop subdivision with curvature control. In: Scheffer, A., Polthier, K. (Eds.), Proceedings of Symposium on Geometry Processing, June 26-28 2006, Cagliari, Italy. ACM Press, pp. 163-172.

Halstead, M., Kass, M., DeRose, T., Aug 1993. Efficient, fair interpolation using Catmull-Clark surfaces. Proceedings of SIGGRAPH 93, 35-44.

Karčiauskas, K., Myles, A., Peters, J., 2006. A $C^{2}$ polar jet subdivision. In: Scheffer, A., Polthier, K. (Eds.), Proceedings of Symposium on Geometry Processing, June 26-28 2006, Cagliari, Italy. ACM Press, pp. 173-180.

Karčiauskas, K., Peters, J., 2007. Bicubic polar subdivision. ACM TOG, xx-xxTo appear.

Karčiauskas, K., Peters, J., Reif, U., July 2004. Shape characterization of subdivision surfaces - case studies. Computer Aided Geometric Design 21 (6), 601-614.

Krishnamurthy, A., Khardekar, R., McMains, S., 2007. Direct evaluation of nurbs curves and surfaces on the gpu. In: SPM '07: Proceedings of the 2007 ACM symposium on Solid and physical modeling. ACM, New York, NY, USA, pp. 
329-334.

Levin, A., 2006. Modified subdivision surfaces with continuous curvature. In: SIGGRAPH '06: ACM SIGGRAPH 2006 Papers. ACM Press, New York, NY, USA, pp. 1035-1040.

Neamtu, M., Pfluger, P. R., 1994. Degenerate polynomial patches of degree 4 and 5 used for geometrically smooth interpolation in $R^{3}$. Computer Aided Geometric Design 11 (4), 451-474.

Peters, J., 1991. Parametrizing singularly to enclose vertices by a smooth parametric surface. In: in: S. MacKay and E. M. Kidd, editors, Graphics Interface '91. Canadian Information Processing Society, Calgary, Alberta, pp. 1-7.

Peters, J., 2000. Patching Catmull-Clark meshes. In: SIGGRAPH '00: Proceedings of the 27th annual conference on Computer graphics and interactive techniques. ACM Press/Addison-Wesley Publishing Co., New York, NY, USA, pp. 255-258, see also: Modifications of PCCM, University of Florida CISE, REP-2001-314.

Peters, J., Reif, U., 2004. Shape characterization of subdivision surfaces: basic principles. Computer Aided Geometric Design 21 (6), 585-599.

Reif, U., 1995a. A note on degenerate triangular Beźier patches. Comput. Aided Geom. Des. 12 (5), 547-550.

Reif, U., 1995b. A unified approach to subdivision algorithms near extraordinary vertices. Computer Aided Geometric Design 12, 153-174.

Reif, U., 1998. TURBS - topologically unrestricted rational $B$-splines. Constructive Approximation. An International Journal for Approximations and Expansions 14 (1), 57-77.

Reif, U., Peters, J., 2005. Structural analysis of subdivision surfaces - a summary. In: et al., K. J. (Ed.), Topics in Multivariate Approximation and Interpolation. Vol. 12. pp. 149-190.

Schaefer, S., Warren, J. D., 2005. On $\mathrm{C}^{2}$ triangle/quad subdivision. ACM Transactions on Graphics 24 (1), 28-36.

Stam, J., Loop, C. T., 2003. Quad/triangle subdivision. Computer Graphics Forum 22 (1), 79-86. 